\title{
Elevated Levels of Serum Pentosidine Are Associated with Dropped Head Syndrome in Older Women
}

Yawara Eguchi ${ }^{1}$, Toru Toyoguchi ${ }^{2}$, Kazuhide Inage ${ }^{3}$, Kazuki Fujimoto ${ }^{3}$, Sumihisa Orita ${ }^{3}$, Kazuyo Yamauchi $^{3}$, Miyako Suzuki ${ }^{3}$, Hirohito Kanamoto ${ }^{3}, \mathrm{Koki} \mathrm{Abe}^{3}$, Masaki Norimoto $^{3}$, Tomotaka Umimura ${ }^{3}$, Masao Koda ${ }^{3}$, Takeo Furuya ${ }^{3}$, Yasuchika Aoki ${ }^{4}$, Kazuhisa Takahashi ${ }^{3}$, Seiji Ohtori ${ }^{3}$

${ }^{1}$ Department of Orthopaedic Surgery, Shimoshizu National Hospital, Yotsukaido, Japan ${ }^{2}$ Department of Orthopaedic Surgery, Chiba Qiball Clinic, Chiba, Japan

${ }^{3}$ Department of Orthopaedic Surgery, Chiba University Graduate School of Medicine, Chiba, Japan

${ }^{4}$ Department of Orthopaedic Surgery, Eastern Chiba Medical Center, Togane, Japan

Study Design: A retrospective observational study was performed.

Purpose: We investigated the prevalence of sarcopenia in dropped head syndrome (DHS), and the relationship between biochemical markers, including major advanced glycation end products (AGEs), pentosidine, and DHS in older women.

Overview of Literature: AGEs have been implicated in the pathogenesis of sarcopenia.

Methods: We studied 13 elderly women with idiopathic DHS (mean age, 77.2 years) and 20 healthy volunteers (mean age, 74.8 years). We used a bioelectrical impedance analyzer to analyze body composition, including appendicular skeletal muscle mass index (SMl; appendicular lean mass [kg]/[height (m) $]^{2}$ ). Cervical sagittal plane alignment, including C2-C7 sagittal vertical axis (C2-C7SVA), C2-C7 angle, and C2 slope (C2S), was measured. Biochemical markers, such as serum and urinary pentosidine, serum homocysteine, 1, 25-dihydroxyvitamin $\mathrm{D}$, and 25-hydroxyvitamin D, were measured. The level of each variable was compared between DHS and controls. The relationship between biochemical markers and DHS was examined.

Results: Sarcopenia (SMI <5.75) was observed at a high prevalence in participants with DHS (77\% compared to $22 \%$ of healthy controls). Height, weight, femoral bone mineral density, appendicular lean mass, total lean mass, and SMI all had significantly lower values in the DHS group. Serum and urinary pentosidine, and serum homocysteine were significantly higher in the DHS group compared to controls. Analysis of cervical alignment revealed a significant positive correlation of serum pentosidine with C2-C7SVA and C2S. Conclusions: Sarcopenia was involved in DHS, and high serum pentosidine levels are associated with severity of DHS in older women.

Keywords: Neck muscles; Sarcopenia; Skeletal muscle; Advanced glycation end products; Homocysteine

Received Apr 27, 2017; Revised Jun 5, 2017; Accepted March 15, 2018

Corresponding author: Yawara Eguchi

Department of Orthopaedic Surgery, Shimoshizu National Hospital, 934-5, Shikawatashi, Yotsukaido, Chiba, 284-0003, Japan

Tel: +81-43-422-2511, Fax: +81-43-421-3007, E-mail: yawara_eguchi@yahoo.co.jp 


\section{Introduction}

Dropped head syndrome (DHS) displays a chin-on-chest deformity because of significant weakness of the neck extensor group [1-6]. DHS can impair quality of life, resulting in restrictions to forward gaze and ambulation, dysphagia, and neck pain. Neck extensor atrophy occurs in a variety of disease backgrounds, including neurologic, neuromuscular, and muscular disorders [1,2]. Among these, because of neck extensor muscle failure of unknown cause, idiopathic DHS is a problem for many elderly patients. There is the possibility for further increase in this condition as society as a whole ages [5].

Sarcopenia is a syndrome characterized by progressive and systemic reduction in skeletal muscle mass. Patients are at high risk of becoming bedridden from a fall, and there is great physical and economic loss in an aging society [7-10]. It is believed that sarcopenia results from inactivity; however, the mechanism is not entirely clear. Decrease in back strength occurs because sarcopenia is believed to contribute to the development of DHS.

Advanced glycation end products (AGEs), such as pentosidine, are the products of nonenzymatic glycation and oxidation of proteins and lipids [11]. They are formed in high concentrations in diabetes, but also in the physiologic organism during aging. AGE crosslinks deteriorate the mechanical and biological functions of bone $[11,12]$.

Recent studies suggest that elevated AGEs levels are independently related to decline in walking abilities, inferior activities of daily living, decreased muscle properties (strength, power, and mass) and increased physical frailty, and may be a contributing risk factor and potential biomarker for decline in motor function [13-15].

Homocysteine interferes with collagen crosslinking. Hyperhomocysteinemia reduces bone strength via a reduction of enzymatic crosslinks and an increase in nonenzymatic crosslinks and pentosidine [12].

Until now, evaluation of DHS has consisted only of local neck magnetic resonance imaging (MRI) $[5,6]$. To our knowledge, no report exists on the involvement of whole body skeletal muscle mass in sarcopenia.

The relationship between AGEs and development of DHS has not been elucidated to our knowledge. We hypothesized that elevated biochemical markers, such as pentosidine and homocysteine, were associated with development of severe DHS. We investigated the prevalence of sarcopenia in DHS, and the relationship between bio- chemical markers, such as pentosidine and DHS, in older women.

\section{Materials and Methods}

\section{Participants}

Study participants were 13 elderly women with idiopathic DHS (mean age, 77.2 years; range, 60-89 years) (Table 1). A total of 20 age- and sex-matched volunteers who underwent osteoporosis examinations (mean age, 74.8 years; range, 62-91 years) served as controls. DHS was defined clinically as a disabling condition in which severe weakness of the neck extensor muscles causes difficulty in lifting the head against gravity, which results in a correctable chin-on-chest deformity (Fig. 1A). Subjects with multiple thoracolumbar compression fractures or a history of spinal surgery were excluded.

\section{Cervical sagittal alignment in dropped head syn- drome}

Radiographs were obtained with the participants in a standing position. Cervical sagittal plane alignment was measured using the $\mathrm{C} 2-\mathrm{C} 7$ sagittal vertical axis (C2C7SVA), C2-C7 angle (C2-C7A), and C2 slope (C2S) (Fig. 1B). Dual-energy X-ray absorptiometry (DXA, Lunar Prodigy; GE Healthcare, Madison, WI, USA) was used to measure bone mineral density (BMD) of the left proximal femur and lumbar spine (L2-L4).

\section{Analysis of skeletal muscle mass}

A multi-frequency bioelectrical impedance analyzer (BIA), the InBody 720 Biospace device (InBody Co. Ltd., Seoul, Korea), was used according to the manufacturer's guidelines. BIA estimates body composition using the difference in conductivity of the various tissues due to the differences in their biological characteristics. Conductivity is proportional to water content (more specifically to electrolytes), and decreases as the cells approach a perfect spherical shape. Adipose tissue is composed of spherical cells and contains relatively little water compared to other tissues, such as muscle; therefore, conductivity decreases as body fat increases. In practice, electrodes are placed at eight precise tactile-points of the body to achieve a multisegmental frequency analysis. A total of 30 impedance 


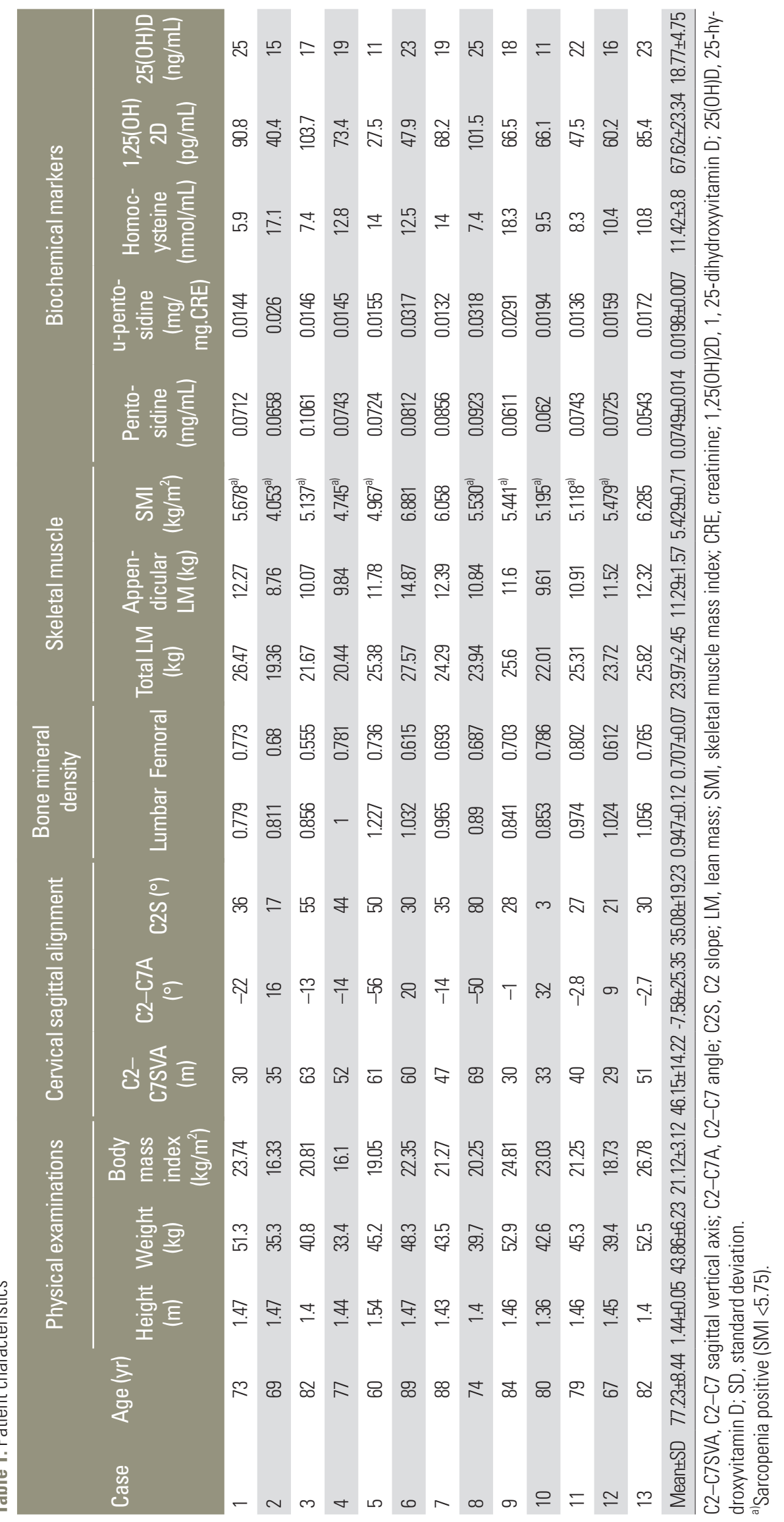




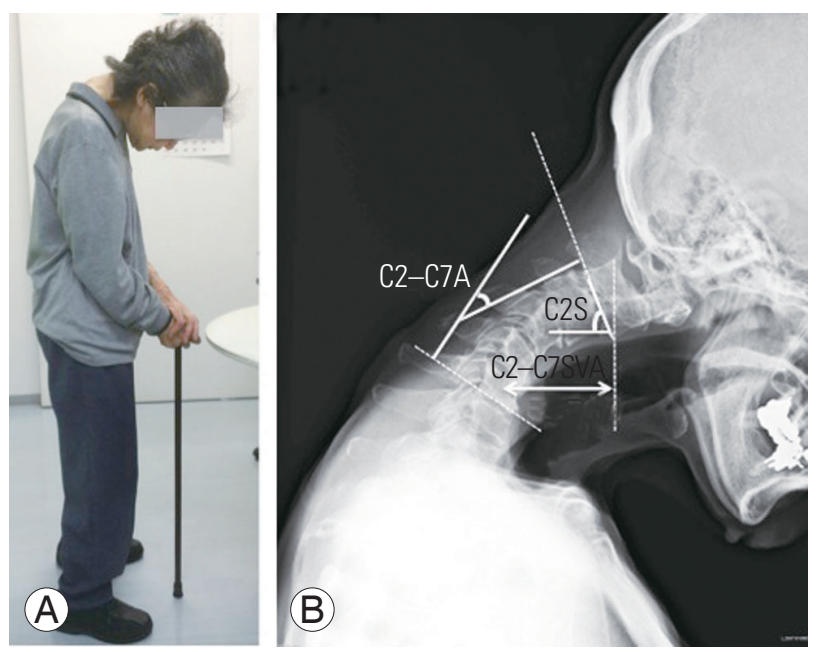

Fig. 1. Photograph (A) and cervical sagittal plane alignment (B) of patient 6 . She cannot raise her head against gravity. C2-C7SVA, C2C7A, and C2S were measured. C2-C7A was negative in the kyphotic direction and positive in the lordotic direction. C2-C7SVA, C2-C7 sagittal vertical axis; C2-C7A, C2-C7 angle; C2S, C2 slope.

measurements were obtained using six different frequencies $(1,5,50,250,500$, and $1,000 \mathrm{kHz})$ for the following five segments of the body: right and left arms, trunk, and right and left legs.

Appendicular skeletal muscle mass was calculated as the sum of skeletal muscle mass in the arms and legs, assuming that mass of lean soft tissue is effectively equivalent to skeletal muscle mass. Appendicular skeletal muscle mass index (SMI) was determined as the sum of arm and leg lean mass $(\mathrm{kg}) /(\text { height }[\mathrm{m}])^{2}$. The diagnosis of sarcopenia among women was defined as appendicular SMI value $<5.75 \mathrm{~kg} / \mathrm{m}^{2}$, determined using sarcopenia normative data [16].

\section{Biochemical markers}

Blood and urinary samples of the participants were collected between the morning and afternoon. The parameters that were measured included serum pentosidine $(\mu \mathrm{g} / \mathrm{mL})$ by enzyme-linked immunoassay (ELISA) and urinary pentosidine $(\mu \mathrm{g} / \mathrm{mg})$; creatinine by ELISA; serum homocysteine $(\mathrm{nmol} / \mathrm{mL})$ by high performance liquid chromatography; 1 , 25-dihydroxyvitamin $\mathrm{D}\left(1,25[\mathrm{OH}]_{2} \mathrm{D}\right)$ $(\mathrm{pg} / \mathrm{mL})$ by radioimmunoassay (RIA); and 25-hydroxyvitamin D (25[OH]D) (ng/mL) by RIA. Serum and urinary pentosidine were measured by the Fushimi Pharmaceutical Co. (Kagawa, Japan); homocysteine and $1,25(\mathrm{OH})_{2} \mathrm{D}$ and $25(\mathrm{OH}) \mathrm{D}$ were measured by SRL Inc. (Tokyo, Japan); and 25(OH)D was measured by the Health Sciences Research Institute East Japan Co. (Saitama, Japan).

\section{Statistical analyses}

We measured height, weight, body mass index (BMI), $\mathrm{BMD}$, appendicular lean mass, total lean mass, SMI, sarcopenia prevalence, and biochemical markers in participants in both groups (Table 2). We investigated the correlation between appendicular SMI or biochemical markers and cervical sagittal alignment (Fig. 2).

StatView software (ver. 5.0; StatView Inc., Nesbit, MS, USA) was used to perform statistical analyses. For each variable, differences between groups were evaluated using an unpaired $t$-test. Differences in the prevalence of sarcopenia between both groups were evaluated using a $\chi^{2}$ test.

Pearson correlation coefficients were calculated to determine the correlation between appendicular SMI or biochemical markers, including serum pentosidine and homocysteine and spinal variables. All data are expressed as the mean \pm standard deviation. A $p<0.05$ was considered significant.

\section{Ethics and consent to participate}

We declare that all protocols involving humans have been approved by the Shimoshizu National Hospital (IRB approval no., H26'-6) and have been performed in accordance with the ethical standards laid down in the 1964 Declaration of Helsinki and its later amendments. We declare that all participants provided written informed consent before their inclusion in this study.

\section{Results}

\section{Physical examinations}

Height, weight, and BMI were significantly lower in patients in the DHS group versus controls (Table 2): $1.44 \pm 0.05$ versus $1.52 \pm 0.05(p<0.001), 43.86 \pm 6.23 \mathrm{~kg}$ versus $54.63 \pm 6.12 \mathrm{~kg}(p<0.001)$, and $21.12 \pm 3.12 \mathrm{~kg} / \mathrm{m}^{2}$ versus $23.60 \pm 3.00 \mathrm{~kg} / \mathrm{m}^{2}$, respectively $(p<0.05)$.

\section{Cervical sagittal alignments in dropped head syndrome}

Cervical spine parameters for the DHS group were C2C7SVA, $46.15 \pm 14.22 \mathrm{~mm}$; $\mathrm{C} 2-\mathrm{C} 7 \mathrm{~A},-7.58^{\circ} \pm 25.35^{\circ}$; and 
Table 2. Physical examination, BMD, skeletal mass, and biochemical markers in the patient participants with DHS and healthy volunteers

\begin{tabular}{|c|c|c|c|c|}
\hline Variable & Normal range & DHS & Control & $p$-value \\
\hline \multicolumn{5}{|l|}{ Physical examinations } \\
\hline Age (yr) & & $77.23 \pm 8.44$ & $74.85 \pm 7.08$ & 0.388 \\
\hline Height (m) & & $1.44 \pm 0.05$ & $1.52 \pm 0.05$ & 0.000044 \\
\hline Weight (kg) & & $43.86 \pm 6.23$ & $54.63 \pm 6.12$ & 0.000028 \\
\hline Body mass index $\left(\mathrm{kg} / \mathrm{m}^{2}\right)$ & & $21.12 \pm 3.12$ & $23.60 \pm 3.00$ & 0.0295 \\
\hline \multicolumn{5}{|l|}{ BMD } \\
\hline Spine $\left(\mathrm{g} / \mathrm{cm}^{2}\right)$ & & $0.947 \pm 0.12$ & $1.043 \pm 0.16$ & 0.074 \\
\hline Femoral $\left(\mathrm{g} / \mathrm{cm}^{2}\right)$ & & $0.707 \pm 0.07$ & $0.814 \pm 0.10$ & 0.003 \\
\hline \multicolumn{5}{|l|}{ Skeletal muscle } \\
\hline Total lean mass (kg) & & $23.97 \pm 2.45$ & $30.29 \pm 2.81$ & 0.00000002 \\
\hline Appendicular lean mass $(\mathrm{kg})$ & & $11.29 \pm 1.57$ & $14.30 \pm 1.63$ & 0.000001 \\
\hline Skeletal muscle mass index $\left(\mathrm{kg} / \mathrm{m}^{2}\right)$ & $>5.75$ & $5.429 \pm 0.71$ & $6.152 \pm 0.56$ & 0.0027 \\
\hline Sarcopenia prevalence (\%) & & 76.92 & 22.22 & 0.00071 \\
\hline \multicolumn{5}{|l|}{ Biochemical markers } \\
\hline Pentosidine ( $\mu \mathrm{g} / \mathrm{mL})$ & $0.00915-0.0431$ & $0.075 \pm 0.014$ & $0.055 \pm 0.013$ & 0.0003 \\
\hline u-Pentosidine ( $\mu \mathrm{g} / \mathrm{mg} . \mathrm{CRE})$ & $0.01942-0.0701$ & $0.019 \pm 0.007$ & $0.014 \pm 0.004$ & 0.0143 \\
\hline Homocystein (nmol/mL) & $4.5-15.3$ & $11.42 \pm 3.80$ & $8.69 \pm 2.89$ & 0.031 \\
\hline $1,25(\mathrm{OH}) 2 \mathrm{D}(\mathrm{pg} / \mathrm{mL})$ & $20-60$ & $67.62 \pm 23.34$ & $67.06 \pm 19.33$ & 0.948 \\
\hline 25(OH)D (ng/mL) & $>20$ & $18.77 \pm 4.75$ & $19.11 \pm 5.03$ & 0.991 \\
\hline
\end{tabular}

Values are presented as mean \pm standard deviation, unless otherwise stated.

BMD, bone mineral density; DHS, dropped head syndrome; CRE, creatinine; 1,25(OH)2D, 1, 25-dihydroxyvitamin D; 25(OH)D, 25-hydroxyvitamin D.

$\mathrm{C} 2 \mathrm{~S}, 35.08^{\circ} \pm 19.23^{\circ}$, representing advanced anteversion and kyphosis (Table 1).

\section{Bone mineral density}

Spinal and proximal femoral BMD were $0.947 \pm 0.12$ versus $1.043 \pm 0.16(p=0.074)$ and $0.707 \pm 0.074 \mathrm{~g} / \mathrm{cm}^{2}$ versus $0.814 \pm 0.101 \mathrm{~g} / \mathrm{cm}^{2}(p<0.01)$ in the DHS group versus controls, respectively. Proximal femoral BMD was significantly lower in the DHS group compared to controls (Table 2).

\section{Skeletal muscle mass}

Regarding skeletal muscle mass parameters, total lean mass, appendicular lean mass, and SMI were $23.97 \pm 2.45$ $\mathrm{kg}$ versus $30.29 \pm 2.81 \mathrm{~kg}(p<0.001), 11.29 \pm 1.57 \mathrm{~kg}$ versus $14.30 \pm 1.63 \mathrm{~kg}(p<0.001)$, and $5.429 \pm 0.71 \mathrm{~kg} / \mathrm{m}^{2}$ versus $6.152 \pm 0.56 \mathrm{~kg} / \mathrm{m}^{2}(p<0.01)$ in the DHS group versus controls, respectively. The DHS group had significantly lower values for all items. The prevalence of sarcopenia was significantly higher in the DHS group than among matched controls, including 10 of 13 (76.9\%) DHS cases versus four of 20 controls $(22.2 \%, p<0.001)$ (Table 2).

\section{Biochemical markers}

Serum and urinary pentosidine, and serum homocysteine levels were significantly higher in the DHS group compared to the controls: $0.075 \pm 0.014$ versus $0.055 \pm 0.013$ $(p<0.001), 0.019 \pm 0.007 \mu \mathrm{g} / \mathrm{mL}$ versus $0.014 \pm 0.004 \mu \mathrm{g} /$ $\mathrm{mL}(p<0.05)$, and $11.42 \pm 3.80 \mathrm{nmol} / \mathrm{mL}$ versus $8.69 \pm 2.89$ $\mathrm{nmol} / \mathrm{mL}(p<0.05)$, respectively. No other variables were significantly different between the groups (Table 2).

\section{Correlation between appendicular skeletal muscle mass index or biochemical markers and cervical alignment}

Cervical alignment showed a significant positive correlation of serum pentosidine with C2-C7SVA $(r=0.623$, $p<0.05)$ and C2S $(r=0.668, p<0.05)$, but no correlation with C2-C7A ( $r=-0.37, p=0.208$ ) (Fig. 2). We found no correlation of any spinal alignment with urinary pento- 

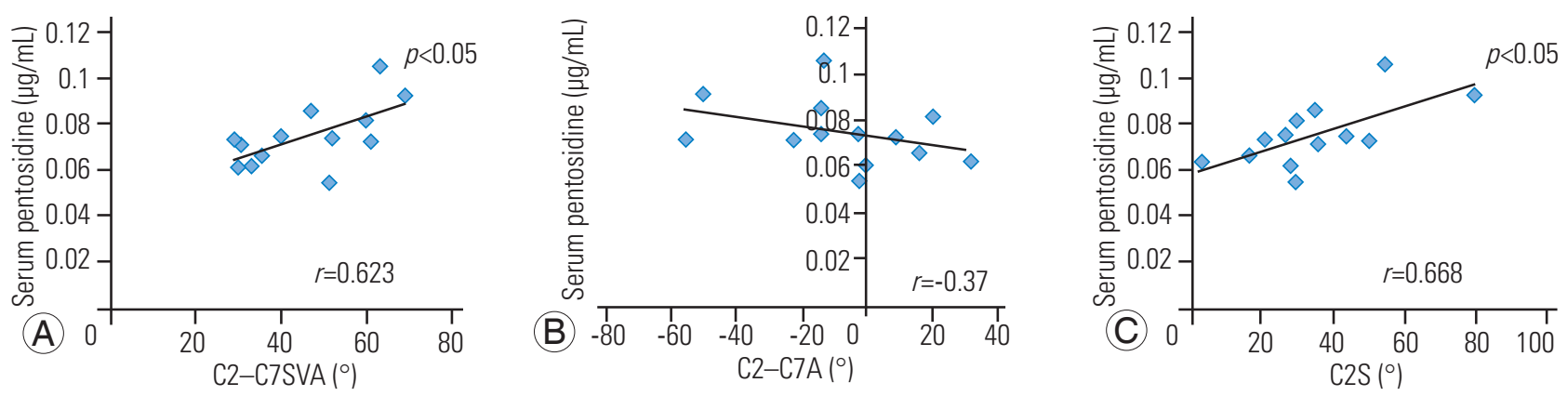

Fig. 2. (A-C) Correlation between serum pentosidine and cervical sagittal alignment. There was a significant positive correlation of serum pentosidine with C2-C7SVA and C2S, but no correlation with C2-C7A. C2-C7SVA, C2-C7 sagittal vertical axis; C2S, C2 slope; C2-C7A, C2-C7 angle.

sidine (C2-C7SVA: $r=0.178, p=0.561$; C2-C7A: $r=0.134$, $p=0.663$; and C2S: $r=0.129, p=0.673)$, serum homocysteine (C2-C7SVA: $r=-0.19, p=0.537$; C2-C7A: $r=0.19$, $p=0.534$; and C2S: $r=-0.3, p=0.311)$, and appendicular SMI (C2-C7SVA: $r=0.218, p=0.474$; C2-C7A: $r=0.085$, $p=0.783$; and C2S: $r=-0.05, p=0.871$ ).

\section{Discussion}

DHS is comprised of a group of disorders associated with the chin-on-chest deformity because of marked weakness of the neck extensor muscles in the setting of a wide variety of diseases, including neurologic, neuromuscular, and muscular disorders [1-6], Parkinson disease [3], multiple system atrophy [4], amyotrophic lateral sclerosis, and isolated neck extensor myopathy (INEM). Katz et al. [5] reported on DHS due to cervical extensor muscle weakness of unknown origin in INEM and proposed an isolated myopathy that occurs because of nonspecific inflammation in the extensor muscle as a result of continued abnormal posture. This is observed in those aged $>60$ years and is confined mainly to the neck extensor muscles. A subacute process is recognized, which includes weakness of shoulder blade and upper arm muscles, myogenic changes in needle electromyography, and muscle atrophy in MRI; however, these features do not extend to other parts of the body.

Until now, evaluation of DHS has consisted of local MRI examination of spinal muscles alone. To our knowledge, no reports exist regarding the association between skeletal muscle mass and sarcopenia.

Sarcopenia is defined as an age-associated loss of skeletal muscle mass and function, and includes a risk of adverse outcomes, such as physical disability and poor quality of life $[7,8]$. Sarcopenia is common in older individuals, with a reported prevalence in 60 to 70 years old of
$5 \%$ to $13 \%$ [9].

Miyakoshi et al. [17] reported that $20 \%$ of Japanese patients with osteoporosis suffer complications because of sarcopenia, while only $10 \%$ of healthy individuals have sarcopenia. However, to our knowledge no studies have clearly defined the relationship between sarcopenia and DHS.

A large proportion of our patients with DHS had sarcopenia compared to controls (78\% versus 22\%). Muscle mass decrease was noted, not only in the neck muscles, but throughout the entire body. Our study showed that serum and urinary pentosidine, and serum homocysteine levels were higher in older women with DHS compared to controls. Serum pentosidine concentration was correlated positively with the severity of DHS seen radiographically. To our knowledge, this is the first study demonstrating biochemical markers, such as pentosidine and homocysteine in DHS.

AGEs accumulate in various musculoskeletal tissues, such as bone [11,12], intervertebral disc [18,19], and muscle [20] with increasing age and adversely affect the biomechanical properties of such structures. Significantly higher levels of AGEs were reported in patients with osteoporosis, increasing the risk of fractures [21,22].

Alterations in concentrations of pyridinoline and pentosidine collagen crosslinks occur with intervertebral disc aging and degeneration, which may contribute to the loss of disc integrity and have a role in the pathogenesis of the degenerative process, such as degenerative disc disease [18], lumbar spondylosis [23], and scoliosis [19].

High AGE levels are associated with decline in muscle function. Serum pentosidine is an independent risk factor for loss of muscle mass in postmenopausal women with type 2 diabetes [15].

AGEs may have a role in sarcopenia through upregula- 
tion of inflammation and endothelial dysfunction in the microcirculation of skeletal muscle through the receptor for AGEs [24].

Homocysteine interferes with collagen crosslinking. The enzymatic and nonenzymatic crosslink deterioration induced by hyperhomocysteinemia (HHcy) may accelerate bone fragility [12]. Previous studies have shown that $\mathrm{HHcy}$ is associated with muscle weakness and lower body weight. HHcy inhibits satellite cell regenerative capacity and enhances oxidative stress thorough p38 MAPK signaling, proposing a potential risk factor for frailty in the elderly [25].

The pathogenesis of idiopathic DHS has not been elucidated. Our study demonstrated that sarcopenia is associated with DHS and high serum pentosidine levels are associated with severity of DHS in older women, suggesting that AGEs levels are potential biomarkers for progression of DHS. Further studies are needed to clarify the mechanism.

Our study has several limitations. The first is a small number of subjects were investigated, requiring confirmation of our findings in a larger population. Second, we did not conduct comprehensive measurements of whole spine alignment. The study was cross-sectional and not longitudinal. DXA appears to be the most reliable tool to evaluate body composition and is considered the criterion standard in clinical practice. BIA may provide a simpler, portative, and less expensive alternative. BIA has a tendency to overestimate muscle mass compared to DXA; however, agreement between DXA and BIA is high for lean mass arm and for axial lean mass [26]. In the future, results should be compared to DXA measurements of muscle mass. The measurement of muscle mass is limited because BIA does not measure some qualities of muscle tissue, such as fat infiltration and muscle functionality. Moreover, the method underestimates the prevalence of sarcopenia in obese subjects and overestimates the prevalence in lean subjects. We also evaluated only slim Japanese women with low BMI; therefore, the amount of truncal fat was much less likely to affect calculations than it might in a typical Western population. Finally, sometimes there was a more rapid decline in muscle strength relative to muscle mass. However, we did not evaluate muscle strength.

\section{Conclusions}

In conclusion, we examined the prevalence of sarcopenia in idiopathic DHS and the relationship between biochemical markers, such as major AGE, pentosidine, and DHS. Sarcopenia was recognized in $77 \%$ of our participants with DHS compared to $22 \%$ of controls, suggesting that sarcopenia may be involved in causing DHS. Serum and urinary pentosidine, and serum homocysteine levels were significantly higher in the patients with DHS than in the controls. High serum pentosidine levels were associated with severity of DHS in older women. Further studies are needed to clarify the pathogenesis of DHS.

\section{Conflict of Interest}

No potential conflict of interest relevant to this article was reported.

\section{Author Contributions}

YE conducted data collection and data entry, and wrote the manuscript. TT developed data collection. KI and KF participated in the design of the study and performed the statistical analysis. All authors contributed to and approved the final manuscript.

\section{References}

1. Petheram TG, Hourigan PG, Emran IM, Weatherley CR. Dropped head syndrome: a case series and literature review. Spine (Phila Pa 1976) 2008;33:47-51.

2. Martin AR, Reddy R, Fehlings MG. Dropped head syndrome: diagnosis and management. Evid Based Spine Care J 2011;2:41-7.

3. Fujimoto K. Dropped head in Parkinson's disease. J Neurol 2006;253 Suppl 7:VII21-26.

4. Quinn N. Disproportionate antecollis in multiple system atrophy. Lancet 1989;1:844.

5. Katz JS, Wolfe GI, Burns DK, Bryan WW, Fleckenstein JL, Barohn RJ. Isolated neck extensor myopathy: a common cause of dropped head syndrome. Neurology 1996;46:917-21.

6. Gaeta M, Mazziotti S, Toscano A, Rodolico C, Mazzeo A, Blandino A. "Dropped-head" syndrome due to isolated myositis of neck extensor muscles: MRI findings. Skeletal Radiol 2006;35:110-2.

7. Cruz-Jentoft AJ, Landi F, Schneider SM, et al. Prevalence of and interventions for sarcopenia in ageing adults: a systematic review: report of the Internation- 
al Sarcopenia Initiative (EWGSOP and IWGS). Age Ageing 2014;43:748-59.

8. Wu IC, Lin CC, Hsiung CA, et al. Epidemiology of sarcopenia among community-dwelling older adults in Taiwan: a pooled analysis for a broader adoption of sarcopenia assessments. Geriatr Gerontol Int 2014;14 Suppl 1:52-60.

9. Morley JE. Sarcopenia: diagnosis and treatment. J Nutr Health Aging 2008;12:452-6.

10. Sanada K, Miyachi M, Tanimoto M, et al. A crosssectional study of sarcopenia in Japanese men and women: reference values and association with cardiovascular risk factors. Eur J Appl Physiol 2010;110:5765.

11. Saito M, Marumo K. Collagen cross-links as a determinant of bone quality: a possible explanation for bone fragility in aging, osteoporosis, and diabetes mellitus. Osteoporos Int 2010;21:195-214.

12. Saito M, Marumo K, Soshi S, Kida Y, Ushiku C, Shinohara A. Raloxifene ameliorates detrimental enzymatic and nonenzymatic collagen cross-links and bone strength in rabbits with hyperhomocysteinemia. Osteoporos Int 2010;21:655-66.

13. Drenth H, Zuidema S, Bunt S, Bautmans I, van der Schans C, Hobbelen H. The contribution of advanced glycation end product (AGE) accumulation to the decline in motor function. Eur Rev Aging Phys Act 2016;13:3

14. Sun K, Semba RD, Fried LP, Schaumberg DA, Ferrucci L, Varadhan R. Elevated serum carboxymethyllysine, an advanced glycation end product, predicts severe walking disability in older women: the Women's Health and Aging Study I. J Aging Res 2012;2012:586385.

15. Tanaka K, Kanazawa I, Sugimoto T. Elevated serum pentosidine and decreased serum IGF-I levels are associated with loss of muscle mass in postmenopausal women with type 2 diabetes mellitus. Exp Clin Endocrinol Diabetes 2016;124:163-6.

16. Janssen I, Baumgartner RN, Ross R, Rosenberg IH, Roubenoff R. Skeletal muscle cutpoints associated with elevated physical disability risk in older men and women. Am J Epidemiol 2004;159:413-21.

17. Miyakoshi N, Hongo M, Mizutani Y, Shimada Y. Prevalence of sarcopenia in Japanese women with osteopenia and osteoporosis. J Bone Miner Metab 2013;31:556-61.

18. Pokharna HK, Phillips FM. Collagen crosslinks in human lumbar intervertebral disc aging. Spine (Phila Pa 1976) 1998;23:1645-8.

19. Duance VC, Crean JK, Sims TJ, et al. Changes in collagen cross-linking in degenerative disc disease and scoliosis. Spine (Phila Pa 1976) 1998;23:2545-51.

20. Haus JM, Carrithers JA, Trappe SW, Trappe TA. Collagen, cross-linking, and advanced glycation end products in aging human skeletal muscle. J Appl Physiol (1985) 2007;103:2068-76.

21. Vaculík J, Braun M, Dungl P, Pavelka K, Stepan JJ. Serum and bone pentosidine in patients with low impact hip fractures and in patients with advanced osteoarthritis. BMC Musculoskelet Disord 2016;17:308.

22. Uchiyama S, Ikegami S, Kamimura M, et al. Bone strength, skeletal muscle area, and biochemical markers associated with bone metabolism in patients with fragility distal radius fracture. J Osteopor Phys Act 2016;4:167.

23. Chiba D, Wada K, Tanaka T, et al. Serum pentosidine concentration is associated with radiographic severity of lumbar spondylosis in a general Japanese population. J Bone Miner Metab 2017;35:65-72.

24. Payne GW. Effect of inflammation on the aging microcirculation: impact on skeletal muscle blood flow control. Microcirculation 2006;13:343-52.

25. Veeranki S, Lominadze D, Tyagi SC. Hyperhomocysteinemia inhibits satellite cell regenerative capacity through p38 alpha/beta MAPK signaling. Am J Physiol Heart Circ Physiol 2015;309:H325-34.

26. Buckinx F, Reginster JY, Dardenne N, et al. Concordance between muscle mass assessed by bioelectrical impedance analysis and by dual energy X-ray absorptiometry: a cross-sectional study. BMC Musculoskelet Disord 2015;16:60. 